\title{
LA VOZ FEMENINA DE LA TRADICIÓN ORAL EN EL MUNDO ÁRABE: LAS NUEVAS SHEREZADES ${ }^{1}$
}

\author{
Margarita Isabel Asensio Pastor²
}

\section{La voz femenina de la tradición oral en el Mundo Árabe: las nuevas Sherezades}

Resumen: La desigualdad de género de la sociedad patriarcal ha hecho que se ignore la voz de la mujer árabe dentro de ella. En este artículo presentamos los resultados de un análisis que sirvió para determinar en qué posición se encuentra la voz femenina árabe. Para ello nos valimos de un muestreo probabilístico aleatorio entre participantes de varios países árabes tanto occidentales como orientales. Variables tales como el género, la edad, la tipología del cuento y el espacio de narración son las que determinan quién tiene la voz autorizada en la tradición oral dentro de las coordenadas culturales árabes.

Palabras clave: mujer, cuentos, tradición oral, cultura, Mundo Árabe.

\section{Female Voice in Oral Tradition in the Arab World: The New Scheherazades}

Abstract: The inequality of patriarchal society has made the voice of Arab women within it ignored. In this paper we present the results of an analysis that was used to determine the position of the Arab female voice. For this we used a random probabilistic sampling among participants of several countries of the Arab West as well as of the East. Variables such as gender, age, story typology and narrative space are those that determine who has the authoritative voice of oral tradition within Arab cultural coordinates.

Keywords: Woman, stories tales, oral tradition, culture, Arab World.

\section{Introducción}

Todos los pueblos disponen de una herencia narrativa a través de la cual, aprenden, aprehenden y comprenden el mundo, a sí mismos y a su comunidad (Calsamiglia y Tusón 2001: 270). Por tanto, narrar es un instrumento personal, social y cultural en donde el cuento ocupa un lugar privilegiado entre las diferentes manifestaciones narrativas desde temprana edad (Bassols y Torrent 2003). Ahora bien, las manifestaciones artísticas orales y escritas, como manifestaciones humanas y culturales, se diferencian, precisamente, en la forma de transmitirse. En este estudio nos centramos en quién es considerado el depositario activo de la tradición oral en el Mundo Árabe (Sydow 1965), es decir, el individuo que dentro de su grupo social mantiene, reproduce o produce tradición oral en el Mundo Árabe, en concreto, el cuento. La hipótesis de la que partíamos, tras un estudio previo de cuentos de tradición oral palestinos-jordanos y la revisión bibliográfica, es que

\footnotetext{
${ }^{1}$ Fecha de recepción: 02/11/2016.

Fecha de aceptación: 23/12/2016.

${ }^{2}$ Profesora Sustituta Interina de Didáctica de la Lengua y la Literatura, Departamento de Educación, Universidad de Almería; $₫$ masensiop@ual.es.
} 
la mujer árabe ocupa un papel relevante como transmisora de este legado; al mismo tiempo, en su voz y narraciones se sustenta el primer contacto del niño desde el punto de vista educativo y sapiencial, de ahí el título de "nuevas sherezades". Sherezade, a través de la narración de cuentos, consigue poner fin a la matanza airada del sultán Shahriar que, tras ser engañado por su mujer, había decidido esposarse con una virgen y decapitarla al día siguiente alcanzando las tres mil mujeres; esta no solo entretenía al sultán, sino que lo iba dotando de moralidad y amabilidad, en otras palabras, lo educaba. Vemos pues, como su voz femenina termina doblegándolo.

Partimos de la base de que el "Mundo Árabe" implica una amplia extensión territorial pues comprende veintidós países (Segura i Mas 2002), con las consiguientes diferencias idiosincráticas, aunque existe entre ellos una idea de identidad común como nos recuerda Segura i Mas (2002: 14) "estos países comparten mayoritariamente una historia, una lengua, una religión comunes e incluso unas características físicas y climáticas. Eso hace que, a pesar del origen étnico diverso, exista un sentimiento de unidad" es lo que se conoce en árabe como arabidad, al-'urūba (العروبة); para Fanjul (1977: 15), este término está relacionado con lo árabe y hace referencia a su forma de ser y comportamiento, a cuanto implica los más hondos sentimientos y afectos familiares y de clan.

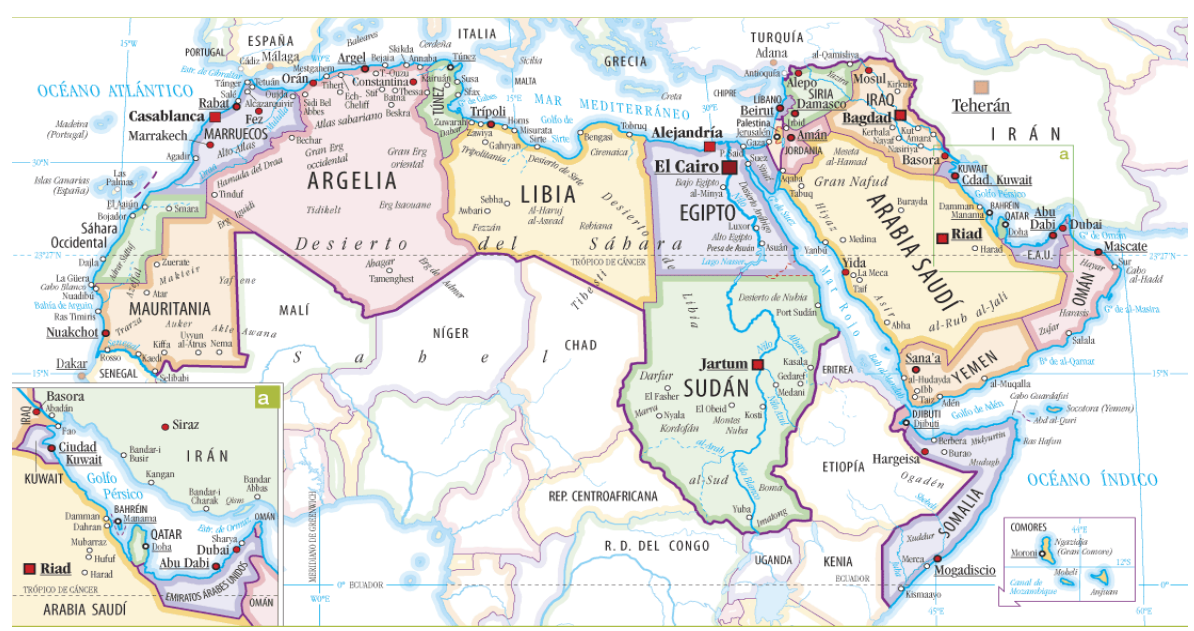

Mapa 1. Mapa de la distribución geográfica de la lengua árabe.

(Fuente: Proyecto Robinson original en cuatricomía elaborado por Paco Arnau y editado en 2006 como anexo cartográfico para Casa Árabe.). 
Nuestro objetivo es, como decíamos, el analizar el comportamiento narrativo de la mujer en el contexto histórico-cultural árabe con respecto a la tradición oral, así como responder a cuestiones tales como qué narra, cuándo narra y dónde lo hace. Una revisión de la bibliografía no manifiesta estudios que puedan dar respuesta a esta cuestión en el marco general árabe, aunque existen algunos relativos a contextos espaciales determinados dentro de él como Rabadán (2000) Piccolo (2015) o Asensio Pastor (2015); no obstante, existen trabajos en los que se atiende al género y a la tradición oral (Kalkun, 2011; Marcussen, 2009; Paloma, 2009 Fishman, 2008; Klinck, 1994; Appadurai 1991; Ortner, 1974).

\section{Método}

El estudio que aquí se presenta parte de un enfoque etnolingüístico centrado en los cuentos de tradición oral de Jordania (incluyendo tanto población transjordana como palestina). A partir de este trabajo se observaron una serie de variables respecto al narrador de cuentos de tradición oral, a la tipología de los cuentos, así como al espacio y tiempo de narración. Ante estas coincidencias se consideró oportuno realizar un estudio de corte cuantitativo que verificara la hipótesis de partida expresada en la introducción de este artículo, la cantidad de la participación femenina en la transmisión narrativa de la tradición oral. Desde esta perspectiva se realizó una nueva investigación, esta vez ampliando el espectro espacial y de población como explicamos en los siguientes epígrafes.

\subsection{Las personas informantes}

La selección de las personas informantes para esta investigación se realizó, en primera instancia, recurriendo a la propia red de amigos, familiares y la red de amigos de estos, por lo que este estudio recoge un muestreo probabilístico aleatorio estratificado por variables género, edad y origen etnonacional (McMillan y Schumacher 2011:140). En palabras de Herrero Muñoz-Cobo (1996: 91), estas variables nos proporcionan 
información relativa al concepto de lengua e identidad social y que, por extensión, en nuestra investigación servían para observar las posibles convergencias y divergencias y entre varias regiones del mundo árabe.

La muestra se compone de 142 participantes. Más de la mitad son mujeres, en concreto, el 54,86 \% sobre el total de las personas encuestadas.

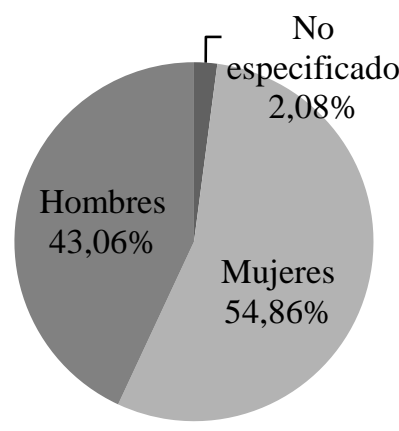

Gráfico 1. Género de los sujetos de la investigación ${ }^{3}$

Los sujetos de la investigación proceden de diversas áreas del Mundo Árabe, excepto de los países del Golfo en una proporción que se observa en el siguiente gráfico:

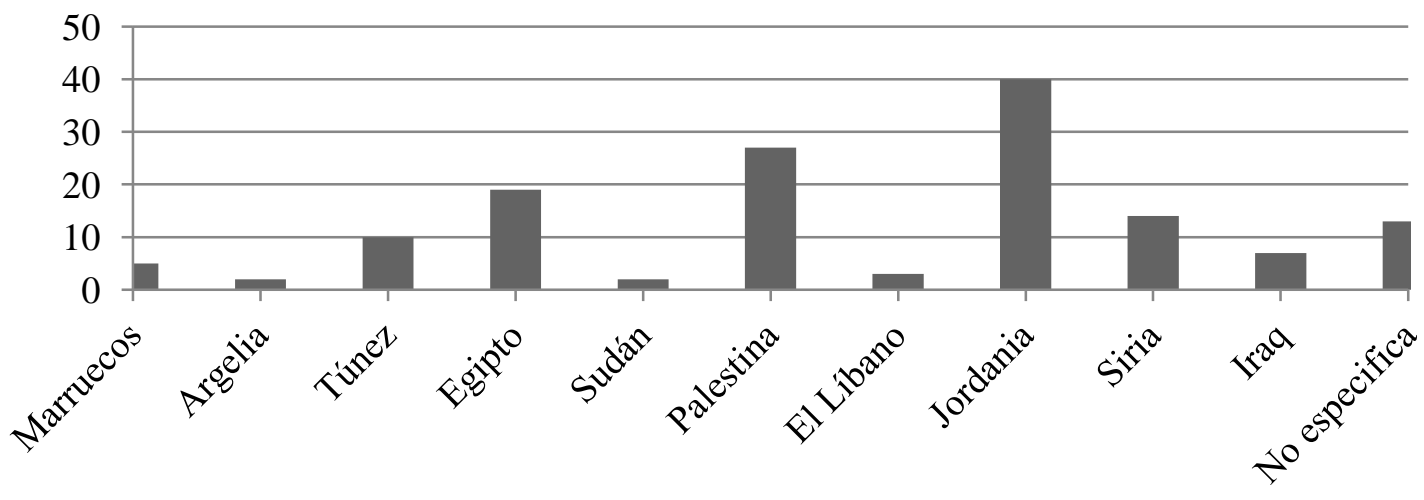

Gráfico 2. País de procedencia de los participantes

Los sujetos de investigación del Oriente árabe (al-mashreq) constituyen el grupo más numeroso con un 78,87\% puesto que lo integran personas procedentes de Egipto, Sudán, Palestina, Jordania, El Líbano, Siria e Iraq; mientras que las muestras del

\footnotetext{
${ }^{3}$ Un 2,08\% de los encuestados no seleccionan la opción de g
} 
Occidente árabe (al-magreb), lo forman participantes de Marruecos, Argelia y Túnez con un porcentaje de presencia del $11,97 \%$.

Tres de los participantes de origen marroquí se definen como bereberes, así como en Egipto, dos de ellos se identifican como griegos egipcios. La población palestina comprende tanto participantes de Gaza como de Cisjordania, especialmente de esta última región. En cuanto al caso de Jordania, el 46,7\% indica su nacimiento en Palestina, si bien disponen actualmente de nacionalidad jordana. Otro dato a tener presente es que gran parte de los participantes de Siria e Iraq no se encuentran actualmente residiendo en sus respectivos países, sino que se encuentran migrados a otros. Por último, existe un grupo en el que dos de los sujetos de la investigación se declararon de origen musulmán; cinco lo hicieron como árabes en general, uno de Oriente Medio y los cinco restantes no proporcionan datos sobre su procedencia.

En cuanto a la edad de los informantes destaca-el grupo comprendido entre los 2535 que representa el 34,51\% de los sujetos de investigación como puede observarse en la gráfica siguiente:

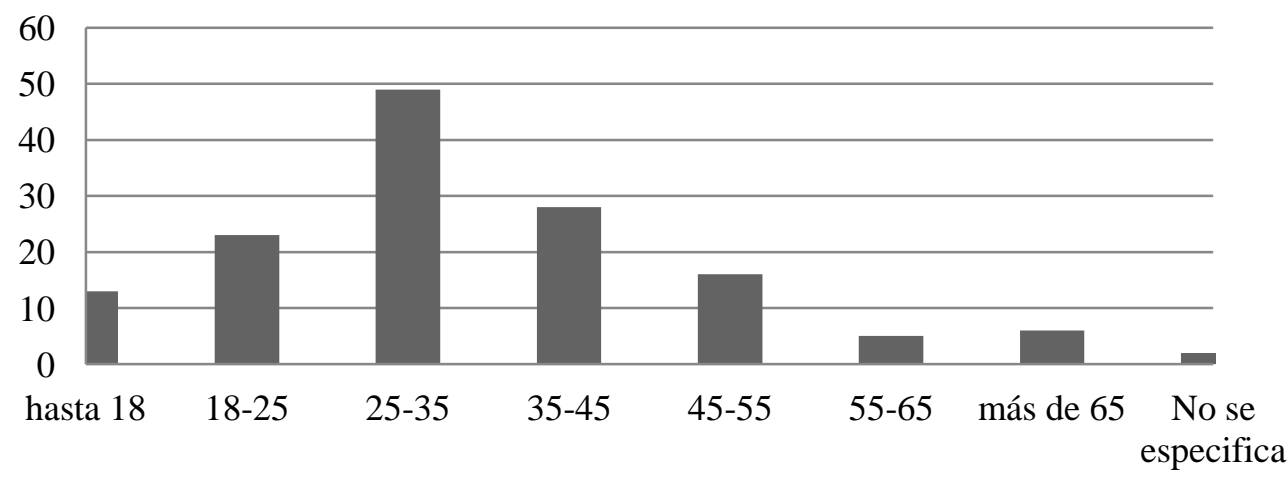

Gráfico 3. Edad de las personas participantes

\subsection{Los instrumentos de investigación}

Los principales instrumentos que se emplearon para la obtención de los datos del cuestionario y la entrevista semiestructurada. En primer lugar, se recurrió a un cuestionario realizado ad hoc en formato digital mediante la aplicación "Formularios de google”. Esta modalidad, sin duda, sirvió para agilizar los trámites de envío y compilación 
de datos. En cuanto a la lengua de redacción de las encuestas se recurrió al español, al inglés y al árabe. El único inconveniente que debimos sortear fue, que para incluir a personas mayores -algunas de ellas analfabetas- dicho cuestionario hubo de hacerse de manera física a través de la propia investigadora o a través de una persona mediadora que, por lo general, era un familiar directo de esta.

En segundo lugar, una vez obtenidos los datos a través del instrumento anterior, se recurrió a la entrevista semiestructurada. En este caso se seleccionó a una serie de personas informantes consideradoas representativas de cada uno de los países por responder al prototipo de depositario activo (Sydow, 1965) de la tradición oral según los términos establecidos en el marco teórico de este trabajo, pero cuya principal característica es la de conservar y transmitir la tradición oral.

\section{Marco teórico}

\subsection{La tradición oral}

La cultura árabe califica las manifestaciones orales de la literaria con el término šafawī / šafahī (شفوي). La característica de esta literatura es el origen popular, su interés por lo hablado, el canto y la escucha, es cutura y se preocupa por ella; además, pervive por la transmisión de generación en generación. Si se perdiese de la memoria, esta desaparecería en contraste con la literatura escrita que fácilmente sobrevive y se transfiere para ser apreciada a lo largo del tiempo, en diferentes épocas y lugares.

Asimismo, la oralidad literaria, a diferencia de la escritura, responde a procedimientos creativos diversos que Pedrosa (2003: 20-21) sintetiza en los siguientes puntos:

- La brevedad puesto que los etnotextos se apoyan en la memoria del transmisor.

- La presencia de fórmulas orales recurrentes y que, en ocasiones, funcionan como apoyos mnemotécnicos.

- El ritmo de los elementos en la disposición del discurso como entonación, modulación de la voz, la musicalidad, entre otros.

- El empleo de interpelaciones o fórmulas dirigidas al oyente/auditorio para captar su atención y hacerlo partícipe de la historia. 
Así pues, de acuerdo con Zumthor (1991: 33-34), hemos de hablar de una "poética específica oral"; esta se sustenta sobre cinco fases que la hacen diferir de la producción de textos escritos y que son la producción, la transmisión, la recepción, la conservación y la repetición. Cada uno de estos criterios responde a dos conceptos relacionados con el etnomaterial: la transmisión y la tradición.

Según esta concepción poética propuesta por Zumthor, la actuación narrativa, performance- la transmisión oral requiere de la presencia en un "aquí y ahora" de narrador/a y un/a oyente (o auditorio) pues es una acción compleja por la que un mensaje poético es simultáneamente transmitido y percibido en este aquí y ahora y en el que coinciden dos ejes de la comunicación social: el que une locutor/a y autor/a, y aquel por el que se unen situaciones y tradición. La persona que actua como transmisor/a se vale, asimismo, de una serie de elementos no verbales y paralingüísticos que, o bien acompañan al texto o lo sustituyen, como la gesticulación, proximidad física, etc. y que sirven para la recepción e interpretación del texto.

Por otro lado, la literatura de tradición oral congrega, al igual que la escrita, diversos géneros (Gómez López y Pedrosa 2003); en el caso del narrativo Simonsen (1984) establece una división en función de la actitud del relato, la forma en la que se presenta, el tipo de protagonista y la función social de este por lo que el cuento lo entiende como un tipo de relato ficcional en prosa, cuyos protagonistas son seres humanos, seres sobrenaturales o animales personificados con la función social de entretener. No obstante, como se ha podido demostrar en trabajos como el de Asensio Pastor (2015) relativo a los cuentos jordanos de tradición oral, en muchos de estos cuentos subyace una función moralizante y pedagógica, especialmente, aquellos que proceden de la población nómadabeduina.

A modo de resumen, en el siguiente cuadro sintetizamos las principales ideas que caracterizan al hecho narrativo de los cuentos de tradición oral: 


\begin{tabular}{|l|l|}
\hline \multirow{4}{*}{ Transmisión oral } & Presencia física de interlocutores (narrador/a -oyente/auditorio) \\
\cline { 2 - 2 } & Comportamiento comunicativo que requiere de los sentidos \\
\cline { 2 - 2 } & $\begin{array}{l}\text { Rápida y espontánea secuencia entre pensamiento y enunciación: aunque } \\
\text { con un esquema hipercodificado. }\end{array}$ \\
\cline { 2 - 2 } & La memoria como soporte de la información \\
\hline
\end{tabular}

Cuadro 1. Elementos de la transmisión cuentística

\subsection{La tradición oral árabe}

La literatura de tradición oral ha estado siempre presente a lo largo del tiempo en el Mundo Árabe. De hecho, la poesía ŷahilī -referida a la época anterior a la llegada del Islam-, como afirma Adonis (1997:123) surge como algo que se oye y no se lee, como cántico y no como escritura. La literatura considerada popular en el Mundo Árabe supone acercarse, por un lado, a la oralidad y a las variedades dialectales y, por otro lado, a los valores culturales que comporta cada una.

Como explica Allen (2000), entre las manifestaciones literarias orales en el Mundo Árabe historias como la de Maŷnūn Layla, una pareja mítica de amantes para la tradición árabe, las guerras tribales recogidas en lo que se conoce como los ayyām al'arab -epopeyas o gestas- o la sīra -la biografía del Profeta Muhammad, así como colecciones de relatos calificados de populares como la historia de los Banī Hilāl ${ }^{4}$-; las historias jocosas del pícaro Ŷoha o la célebre compilación Las mil y una noches que para el-Shamy (1990) comprende gran parte de los relatos de literatura tradicional árabe. Dando un salto temporal en este recorrido diacrónico, la Nahḍa ("Renacimiento o "Despertar Árabe") que se produce a mediados del siglo XIX y principios del XX (Martínez Montávez, 1994), supuso la eclosión de los estudios sobre tradición oral. Esto demuestra, por un lado, que la tradición oral árabe ha sobrevivido, al margen o en paralelo, de lo que se considera la "cultura oficial árabe" basada en la escritura y, por otro, que, si ha superado el paso del tiempo, se debe al interés social que despierta.

4La Sīra de los Banī Hilāl cuenta la historia de un héroe, Abu Zayd al-Hilālī que viajó de Oriente a Occidente, hacia Túnez, conquistando estas tierras para el Islam representa el coraje y la fortaleza (Allen 2000: 173). 
La Nahda fue una toma de conciencia y la revalorización de la herencia folclórica de cada país árabe en el momento de la descolonización del dominio otomano que provocó la urgencia de recuperar las tradiciones y especialmente la cultura oral vehiculada por las lenguas vernáculas, como apunta Fanjul (1977: 49):

"modernamente, y en países subdesarrollados, el interés por el folklore reviste un cierto carácter revolucionario que en los países árabes había sido sistemáticamente marginado por evadirse, a la vez, de la cultura clásica o de los modelos extranjerizantes. El surgimiento a flor de agua del folklore no es tanto el del individuo como el de las clases desdeñadas. Porque aunque no exista una prohibición formal de publicar obras en árabe vulgar (el único que utiliza la literatura popular), sí existe una "selección por el descrédito".

Se vuelve imperativo, así, definir la identidad nacional sin renunciar a la "arabidad" compartida. Por tanto, este volver la vista a lo tradicional suponía en la práctica una revalorización de lo popular y del folclore y las lenguas orales, al ser consideradas formas menos afectadas, más naturales. Para Badawi (1992: 269), la incursión de lo tradicional y popular en la esfera literaria implica la ruptura de la barrera entre los dos registros lingüísticos; se rompen las barreras entre lo coloquial y el árabe clásico o estándar y, al mismo tiempo, este último se ve enriquecido por las aportaciones de la tradición oral y adoptando sus técnicas.

\section{La figura narradora en la tradición oral árabe}

Nuestro cuestionario se centró en las siguientes cuestiones que, además, corresponden al orden expositivo que aquí desarrollaremos:

- ¿Quién narra los cuentos de tradición oral?

- ¿Cuál es la tipología de estos cuentos?

- ¿Cuándo se suelen contar/ se les solía contar?

- ¿Dónde se suelen / se solían contar?

Una serie de cuestiones son relevantes en tanto que aluden al depositario activo de la tradición oral actualmente en el Mundo Árabe: 
La primera es que la narración de cuentos de tradición oral actualmente apenas se relaciona con una figura profesional, por tanto, no podemos hablar de un depositario activo de la tradición oral profesional y que en árabe recibe el nombre de hakawātī (حكو اتي) -en la parte oriental- o $r \bar{a} w \bar{l}$ (راوي) -en la parte occidental. La mayoría de las personas encuestadas asocian la narración de los cuentos de tradición oral con uno o varios miembros familiares o lo relacionan con la escuela y el docente. De hecho, la presencia del hakawātī lo encontramos solo en un encuestado jordano de más de 50 años y tres sirios procedentes de la ciudad de Damasco donde, antes de estallar la guerra, era posible oír a un hakawātī en el café al Nofara. La segunda es que el cuento de tradición oral se observa como un tipo discursivo predominantemente femenino. En este sentido, las narradoras por excelencia son las madres con un $29,38 \%$ de forma aislada y junto con el padre en un 5,67\%, a estas le siguen las abuelas con un 31,44\% -al respecto hemos de especificar que algunas de las personas participantes indican si se trata de abuela materna o paterna- y, por último, se sitúan a las tías con un 7,73\%. Frente a la parte masculina que no llega a alcanzar el $10 \%$ del total. Por otro lado, dentro de este apartado hemos de puntualizar que cuando los participantes se refieren a la escuela, suelen referirse principalmente a la Profesora de Primaria.

La tercera, apoyando el carácter femenino de la narración de tradición oral, se sitúa la diferencia entre realidad y ficción del relato. Cuando este es del género cuento o leyenda, entre otros se considera más propio de la mujer, mientras que anécdotas o historietas más vinculadas a la realidad se asocian al hombre.

\footnotetext{
5 La palabra hakawātī (حكواتي) significa "narrador popular", es decir, "cuentacuentos"; proviene de la raíz hawà (حكى) que se puede traduce como “contar, narrar, imitar y transmitir” (Cortés, 1996: 257-258). Su existencia está atestiguada desde el siglo X por Ibn al-Nadim o al-Mas`udi, según expone Qabbān̄̄ (1986), en el mundo árabe incluido el otomano.
} 


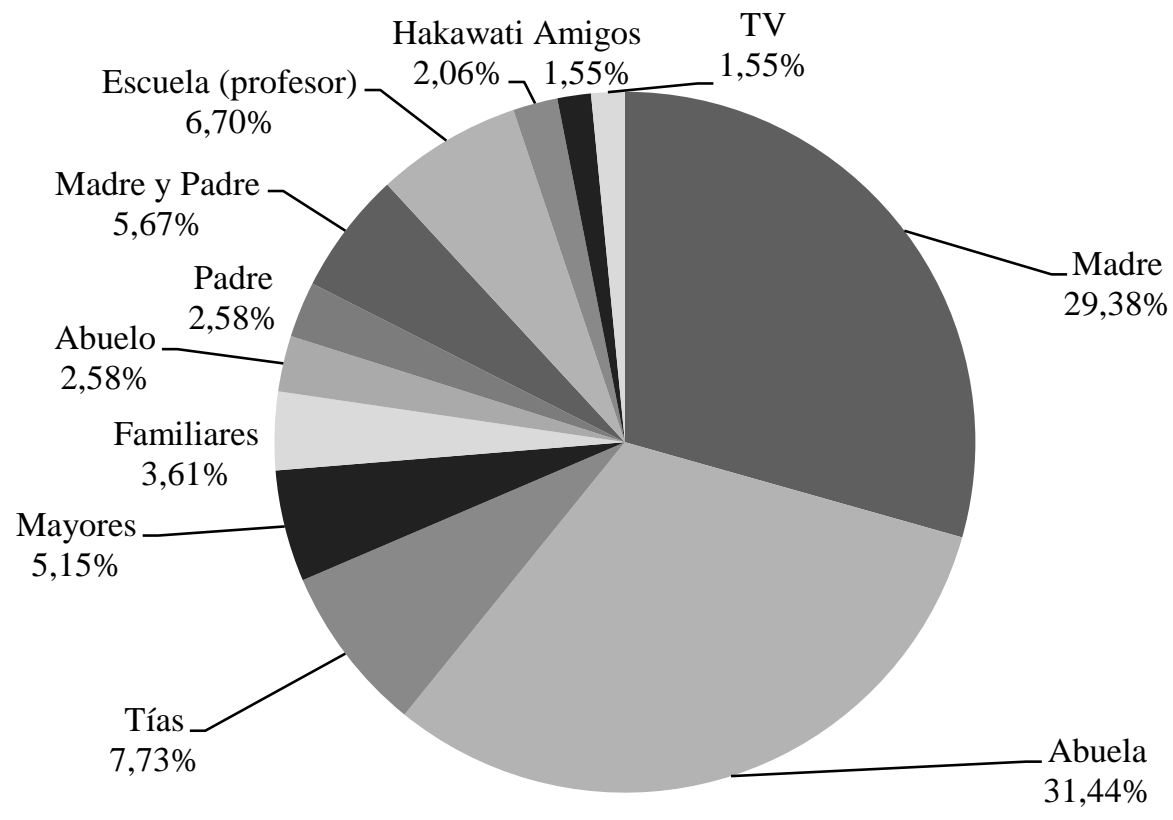

Gráfico 4. Depositarios/as activos/as y narradores de tradición oral.

Además de la cuestión de género, hemos de destacar la importancia que adquiere la edad dentro de los sujetos de la investigación ya que cerca del 46,9\% de las personas narradoras son miembros de edad avanzada, tanto dentro de la familia, como en el contexto social en general, como queda patente en el hecho de que varios sujetos se refieran a ellos expresamente como al-kibār (los mayores), siguiendo a Bassiouney (2009) y Herrero Muñoz-Cobo (1996), la mujer árabe gana estatus.

Por último, si bien existen estudios como el de Appadurai (1991) en el que se asocia a la mujer a la tradición oral, lo hace debido a una situación de analfabetismo. En la investigación de Asensio Pastor (2015), no se muestra como característica del depositario activo de la tradición oral el analfabetismo, aunque a mayor edad del informante -sobre todo femenino- menor grado formativo. No obstante, en esta investigación, los datos no son concluyentes a este respecto, si bien lo es el espacio de narración (privado-público) y la asociación de este con las personas informantes. 


\subsection{La tipología de los cuentos}

La tipología de los cuentos es, como no podía ser de otra manera, de corte ficcional, aunque la clasificación que ofrecemos atiende a lo que los participantes expresaron en las encuestas; así pues, encontramos relatos de fantasía o maravilla, de miedo y aventuras. Asimismo, aparecen cuentos ligados a la realidad del grupo en el que destacan dos cuestiones: historia del país especialmente recurrente entre la población palestina o de origen palestino y relatos sobre la revolución y la resistencia tanto entre los grupos anteriores como en los tunecinos.

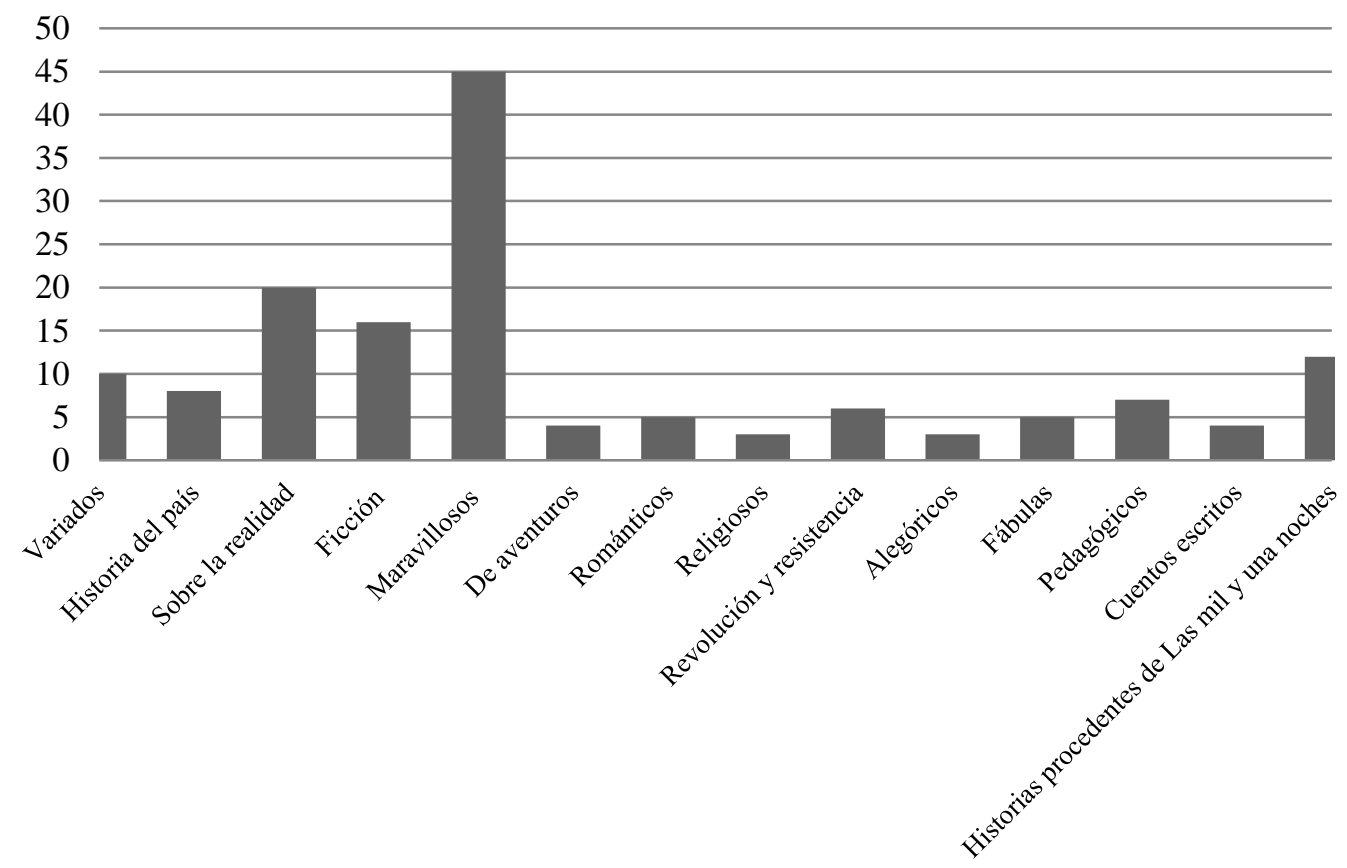

Gráfico 5. Tipología de cuentos de tradición oral

A tenor de esta clasificación, se percibe una tendencia entre el sector de la población sedentaria y urbana a los cuentos de corte maravilloso (jurafiyya) con un $30,40 \%$ junto con los relatos procedentes de Las mil y una noches $(8,11 \%)$. Asimismo, se incluirían esta categoría de ficción el resto de cuentos de aventuras, románticos, alegóricos o fábulas, así como pedagógicos, los cuales pueden también contener 
elementos maravillosos como se pudo observar en el estudio mencionado sobre cuentos jordanos de tradición oral (Asensio Pastor 2015). Así pues, una de las ideas que nos vuelven a acercar a la mujer como la voz autorizada de los cuentos folclóricos es el carácter ficcional de estos. Como expone Rabadán Carrascosa (2003: 52) al referirse a los cuentos maravillosos en Palestina considera que "es poco frecuente que los hombres narren este tipo de cuento indiscutiblemente relacionado con la patraña y la ficción (...)" por lo que los define como "un arte de mujeres".

Por otro lado, se observan dos cuestiones decisivas para la tipología de los cuentos y que son el origen y estilo de vida de las persons participantes y la situación histórica del país de procedencia. Con respecto a los estilos de vida que conviven actualmente en los países de procedencia de los encuestados podemos establecer la siguiente clasificación:

- La población sedentaria distribuida en dos tipos de asentamiento: uno urbano y el otro rural.

- La población semi-nómada que se desplaza dos veces al año a zonas acotadas para el pastoreo.

- La población beduina, es decir, el sector de la población nómada.

Algunos de los participantes especifican su origen beduino como sucede con un iraquí y tres jordanos. Precisamente es en estos donde los cuentos que se mencionan son de corte pedagógico y que son relatos que ayudan a entender cuestiones meteorológicas o, incluso, valores relacionados precisamente con este modo de vida nómada como es la generosidad para con el viajero o la viajera. En esta tipología sobre sale el carácter real de los relatos frente a lo ficcional debido, por tanto, a las necesidades derivadas de lo inhóspito del espacio vital y la ausencia total o parcial de rudimentos de herramientas lectoescritoras depositando en la oralidad, por tanto, la sabiduría popular. Por último, los relatos sobre la historia nacional constituyen un un $9,46 \%$ de los tipos de cuentos.

En definitiva, el carácter ficcional se asocia a la mujer y el real al hombre. Sin embargo, los cuentos de ficción contienen un alto componente moralizante y educativo 
que los convierten en la primera forma de instrucción y donde la mujer es la que tiene la voz autorizada.

\subsection{El espacio y el tiempo de narración}

Consideramos oportuno introducir, en los cuestionarios, el espacio de narración lo que, por otro lado, determinó el tiempo en el que los participantes solían oír las narraciones. Con respecto al espacio, como señala Herrero Muñoz-Cobo (1996, 2016), la árabe es una sociedad tradicional que tiende a delimitar los espacios: público ('àmm) asociado a lo masculino y privado (jāsș ), ligado a lo femenino. En la gráfica que sigue, sobresale el hogar como el espacio de narración del cuento tradicional; al respecto, (algunos de las personas participantes señalan si se trata del hogar de su núcleo familiar propiamente dicho o el de abuelos y abuelas). Por otro lado, también se especifica si la narración se produce en el salón $(19,18 \%)$, el dormitorio $(78,08 \%)$ u otros lugares como la azotea $(1,37 \%)$ o el jardín $(1,37 \%)$ y la escuela. En el espacio público, la calle se produce un 2,11\% de las narraciones lo que es lógico si tenemos en cuenta la ausencia de una figura profesionalizada en la transmisión de relatos.

Como expusimos en el epígrafe relativo al tipo de informante, el espacio de narración viene condicionado por el género de dicho informante. Así, lo público es propicio para el hombre y el privado, para la mujer revelando un hecho fundamental, en relación con la tipología y es el carácter moralizante y educativo de estos para la formación en valores del infante. 


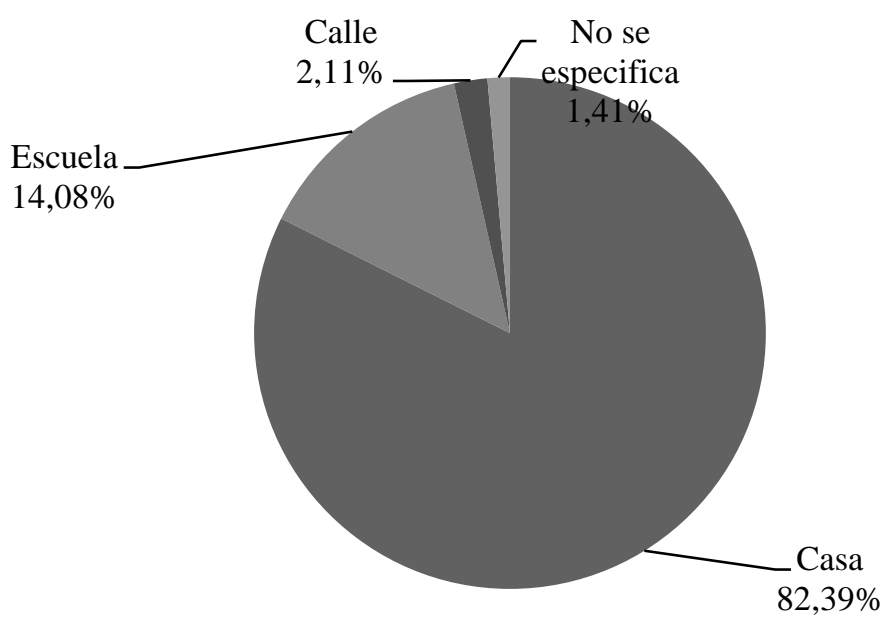

Gráfico 6. Espacios de narración

En relación con el tiempo, la información que se infiere es que se trata de un tipo de narración asociada con la tarde y la noche, inclusive -como es obvio- para quienes determinan como informantes a la televisión. Además, un 15,67\% indica que este tipo de narraciones se suelen dar durante las reuniones familiares, entendiendo la familia desde el prisma de familia extensa (Herrero Muñoz-Cobo, 2017) paradigma que sigue vigente en algunos zonas como es el caso de Jordania.

\section{Conclusiones}

En el título de este artículo hacíamos referencia a la figura de Sherezade, ya que consideramos que la voz de las mujeres en el Mundo Árabe, al igual que este arquetipo literario, precisamente de la tradición oral responde, a nuestro entender, a la idea que subyace bajo este nombre, la búsqueda femenina de la liberación de su voz a través de la sabiduría y como es, en la voz de esta, donde se asienta la base social. Esta sabiduría, por otra parte, hemos de concebirla dentro de los parámetros de lo popular, de la transmisión de boca a oído de historias para aprender a ser uno mismo y, al mismo tiempo, encontrar un lugar dentro de la sociedad.

Asimismo, con todo lo expuesto anteriormente podemos concluir que la narración de cuentos de tradición oral, en gran parte del Mundo Árabe tiene voz de mujer que, por 
tanto, es la voz autorizada y depositaria activa de la tradición oral árabe. El hecho de que la ficción y fantasía es el ámbito propio de mujeres, mientras que lo real lo es del hombre. Se ha demostrado en trabajos de tipo cualitativo de Asensio Pastor (2015), Piccolo (2015) o Rabadán Carrascosa (2003).

Por otro lado, las diferencias de estilo y origen de vida de quienes participan no influyen para que la mujer pierda el estatus de narradora de cuentos; ahora bien, la sedentarización o la vida beduina sí inciden sobre la tipología de los cuentos acercándolos o alejándolos de la realidad.

\section{Referencias bibliográficas}

Adonis. 1997. Poesía y poética árabes. Madrid: Ediciones del Oriente y del Mediterráneo.

Allen, R. M. A. 2000. An Introduction to Arabic Literature. Cambridge: Cambridge University Press.

Appadurai, A. 1991. "Afterword” en Arjun Appadurai, Frank J. Korom, y M. A. Mills (Eds.), Gender, Genre and Power in South Asian Expressive traditions. (South Asia Seminar Series, general ed. Patrick Mullen) Philadelphia: University of Pennsylvania Press: 467-476.

Asensio Pastor, M. I. 2015. Cuentos jordanos de tradición oral: estudio etnolingüístico y aplicaciones didácticas. Almería: Universidad de Almería [tesis inédita].

Badawi, M. M. 1992. Modern Arabic Literature. Cambridge: Cambridge University Press.

Bassiouney, R. 2009. Arabic Sociolinguistics. Edimburgo: Edinburgh University Press.

Bassols, M. y Torrent, A. M. 2003. Modelos textuales. Teoría y práctica. Barcelona: Octaedro.

Calsamiglia Blancafort, H. y Tusón Valls, A. 2001. Las cosas del decir. Manual de análisis del discurso. Barcelona: Ariel.

Cortés, J. 1996. Diccionario de árabe culto moderno. Árabe-español Madrid: Gredos.

El-Shamy, H. 1990. Oral Traditional Tales and the Thousand Nights and a Night: The Demographic Factor. Bloomington: University of Indiana.

Fanjul, S. 1977. Literatura popular árabe. Madrid: Editorial Nacional. 
Fishman , A. 2008. "Thrênoi to Moirológia: Female Voices of Solitude, Resistance, and Solidarity"

Oral

Tradition,

23/2:

267-295

http://journal.oraltradition.org/files/articles/23ii/07_23.2.pdf [28 mayo 2016].

Gómez López, N. y Pedrosa, J M. (Eds.) 2003. Las voces sin fronteras: didáctica de la literatura oral y la literatura comparada. Almería: Servicio de publicaciones Universidad de Almería.

Herrero Muñoz-Cobo, B. 1996. El árabe marroquí: aproximación sociolingüística. Almería: Universidad de Almería.

Herrero Muñoz-Cobo, B. 2017. Elementos de unidad y pluralidad en el Mundo Árabe. Madrid: Cantarabia.

Kalkun, A. 2011. A Woman's Voice in an Epic: Tracing Gendered Motifs in Anne Vabarna's Peko. http://www.jef.ee/index.php/journal/article/viewFile/28/pdf_27 [28 mayo 2016].

Klinck, Anne L. 1994. Lyric Voice and the Feminine in Some Ancient and Mediaeval Frauenlieder" Florilegium, https://journals.lib.unb.ca/index.php/flor/article/viewFile/18459/20276\%EF\%BF $\% \mathrm{BD} \% \mathrm{C} 3 \% 9 \mathrm{C}$ [28 mayo 2016].

Marcussen, E. 2009. Gender as a Genre in Women's Oral Tradition in India http://www.ibg.uu.se/digitalAssets/139/a_139833-f_marcussen-final-hindi-d.pdf [20 mayo 2016].

Martínez Montávez, P. 1992. Introducción a la literatura árabe moderna. Madrid: Cantarabia.

McMillan, J. H. y Schumacher, S. 2011. Investigación educativa. Madrid: Pearson.Addison Wesley.

Ortner, S. B. 1974. Is Female to Male as Nature Is to Culture? en M. Z. Rosaldo and L. Lamphere (Eds), Woman, Culture, and Society. Stanford, CA: Stanford University Press: 68-87. https://www.uio.no/studier/emner/sv/sai/SOSANT1600/v12/Ortner_Is_female_to _male.pdf [25 mayo 2016]

Rabadán Carrascosa, M. 2003. La jrefiyye Palestina: Literatura, mujer y maravilla. El cuento maravilloso palestino de tradición oral. Estudio y textos. México, D.F. Colegio de México.

Simonsen, M. 1984. Le conte populare. París: Presses Universitarieres de France.

Sydow, C. W. von. 1965. "Folktale Studies and Philology: Some Points of View" en dundes, A. (Ed.) The Study of Folklore. Englenwood Cliffs: Prentice-Hall, 219-242.

Zumthor, P.1991. Introducción a la poesía oral. Madrid: Taurus. 